\title{
A Class of $d$-Dimensional Dirac Operators with a Variable Mass
}

\author{
Asao Arai and Dayantsolmon Dagva \\ Department of Mathematics, Hokkaido University, Sapporo 060-0810, Japan \\ Correspondence should be addressed to Asao Arai; arai@math.sci.hokudai.ac.jp
}

Received 3 May 2013; Accepted 9 June 2013

Academic Editors: S. Ravindran and C. Zhu

Copyright (C) 2013 A. Arai and D. Dagva. This is an open access article distributed under the Creative Commons Attribution License, which permits unrestricted use, distribution, and reproduction in any medium, provided the original work is properly cited.

A class of $d$-dimensional Dirac operators with a variable mass is introduced ( $d \geq 2)$, which includes, as a special case, the 3 dimensional Dirac operator describing the chiral quark soliton model in nuclear physics, and some aspects of it are investigated.

\section{Introduction}

In the chiral quark soliton (CQS) model in nuclear physics (see, e.g., [1] and references therein), a Dirac operator of the following form appears (we use the physical unit system where the speed of light $c$ and $\hbar$, the Planck constant divided by $2 \pi$, are equal to 1$)$ :

$$
H_{\mathrm{CQS}}:=-i \sum_{j=1}^{3} \alpha_{j} D_{j} \otimes 1_{2}+m \beta \otimes 1_{2} e^{i \sum_{j=1}^{3} F \gamma_{5} \otimes \tau_{j} n_{j}}
$$

acting in the tensor product Hilbert space $L^{2}\left(\mathbb{R}^{3} ; \mathbb{C}^{4}\right) \otimes$ $\mathbb{C}^{2}$ of $L^{2}\left(\mathbb{R}^{3} ; \mathbb{C}^{4}\right)$ (the Hilbert space of $\mathbb{C}^{4}$-valued square integrable functions on $\mathbb{R}^{3}$ ) and $\mathbb{C}^{2}$. Here $i$ is the imaginary unit, $\alpha_{1}, \alpha_{2}$, and $\alpha_{3}$ are $4 \times 4$ Hermitian matrices obeying the anticommutation relations

$$
\left\{\alpha_{j}, \alpha_{k}\right\}=2 \delta_{j k} 1_{4} \quad(j, k=1,2,3)
$$

$\left(\{A, B\}:=A B+B A, \delta_{j k}\right.$ is the Kronecker delta and $1_{n}$ denotes the $n \times n$ unit matrix $), D_{j}(j=1,2,3)$ is the generalized partial differential operator in the space variable $x_{j}(\mathbf{x}=$ $\left.\left(x_{1}, x_{2}, x_{3}\right) \in \mathbb{R}^{3}\right), m>0$ denotes the mass of a quark, $\beta$ is a $4 \times 4$ Hermitian matrix satisfying

$$
\begin{gathered}
\left\{\alpha_{j}, \beta\right\}=0 \quad(j=1,2,3), \quad \beta^{2}=1_{4}, \\
\gamma_{5}:=-i \alpha_{1} \alpha_{2} \alpha_{3},
\end{gathered}
$$

$F: \mathbb{R}^{3} \rightarrow \mathbb{R}$ is a function called a profile function, $\tau_{1}, \tau_{2}$, and $\tau_{3}$ are the Pauli matrices, and $n_{j}: \mathbb{R}^{3} \rightarrow \mathbb{R}(j=1,2,3)$ is a Borel measurable function on $\mathbb{R}^{3}$ such that $\sum_{j=1}^{3} n_{j}(\mathbf{x})^{2}=1$ for a.e. (almost everywhere) $\mathbf{x} \in \mathbb{R}^{3}$.

Comparing $H_{\mathrm{CQS}}$ with the usual free Dirac operator with mass, one notes that the term

$$
m(\mathbf{x}):=m e^{i \sum_{j=1}^{3} F(\mathbf{x}) \gamma_{5} \otimes \tau_{j} n_{j}(\mathbf{x})}
$$

corresponds to a mass, although it may depend on the space variable $\mathbf{x}$ in general. Hence, the CQS model may be regarded as a model of a Dirac particle with a variable mass. We also note that $m(\mathbf{x})$ is not a scalar multiple of a constant matrix in general but may be a nontrivial matrix-valued function on $\mathbb{R}^{3}$. This is one of the interesting features of the Dirac operator $H_{\mathrm{CQS}}$. From a general point of view, $m(\mathbf{x})$ is a special case of the mass deformation of the form $m_{L}(\mathbf{x}):=m L(\mathbf{x})$ with $L$ being a mapping from $\mathbb{R}^{3}$ to the set of linear operators on $\mathbb{C}^{4} \otimes$ $\mathbb{C}^{2}$. To our best knowledge, mathematically rigorous analysis on Dirac operators with such a mass deformation seems to be few, although a Dirac operator with a mass given by a scalar function has been studied (e.g., [2]).

In a paper [3], Arai et al. investigated spectral properties of the Dirac operator $H_{\mathrm{CQS}}$. These results have been extended to the case of a generalized CQS (GCQS) model in [4]. Miyao [5] proposed an abstract version of the CQS model and investigated a nonrelativistic limit of it; as an application of the abstract result to the CQS model, a Schrödinger operator with a binding potential was derived.

As is pointed out in [3], under a condition for $n_{j}(\mathbf{x})(j=$ $1,2,3)$, the CQS model has supersymmetry; that is, the Dirac operator $H_{\mathrm{CQS}}$ may be a supercharge of a supersymmetric 
quantum mechanics (e.g., [6, Chapter 5]). This structure is carried over to the GCQS model [4].

In this paper, for each natural number $d \geq 2$, we propose a $d$-dimensional version of the GCQS model and analyze some mathematical aspects of it including supersymmetric ones.

The present paper is organized as follows. We first recall some basic facts in operator theory in Section 2. In Section 3 we introduce a Dirac operator $H$ which may be the Hamiltonian of a $d$-dimensional version of the GCQS model, as mentioned previously. A simple condition for $H$ to be self-adjoint is given. In Section 4 we discuss supersymmetric aspects of $H$. We give a condition for $H$ to be a supercharge of a supersymmetric quantum mechanical model. In that case, ker $H$, the kernel of $H$, describes the supersymmetric states. Hence, it is interesting and important to analyze ker $H$. In Section 5, we prove that, under a condition, ker $H$ is trivial: ker $H=\{0\}$. In the case where $H$ is a supercharge, this means that there is no supersymmetric states; namely, the supersymmetry is spontaneously broken. Section 6 is concerned with a unitary equivalence of $H$ to a gauge theoretic Dirac operator. This may be physically interesting. Using this structure, we find another condition for the kernel of $H$ to be trivial. In Section 7, we identify the essential spectrum of $H$ under a suitable condition. In the last section, we discuss the number of eigenvalues of $H$ in the interval $(-m, m)$ with $m>0$ being a constant.

\section{Preliminaries}

Let $\mathscr{X}$ be a complex Hilbert space with inner product $\langle\cdot, \cdot\rangle_{X}$ (linear in the second variable) and norm $\|\cdot\|_{\mathscr{X}}$ (we sometimes omit the subscript $\mathscr{X}$ if there is no danger of confusion). For a linear operator $A$ on $\mathscr{X}$, we denote its domain by $D(A)$. If $A$ is densely defined, its adjoint is denoted by $A^{*}$. For linear operators $A$ and $B$ on $\mathscr{X}, A \subset B$ means that $B$ is an extension of $A$, that is, $D(A) \subset D(B)$ and $A \psi=B \psi$, for all $\psi \in D(A)$.

We denote by $\mathfrak{B}(\mathscr{X})$ the set of everywhere defined bounded linear operators on $\mathscr{X}$. For $T \in \mathfrak{B}(\mathscr{X})$, we denote the operator norm of $T$ by $\|T\|$.

Definition 1. Let $A$ and $B$ be self-adjoint operators on $\mathscr{X}$.

(i) $A$ and $B$ are said to strongly commute if their spectral measures commute.

(ii) $A$ and $B$ are said to strongly anticommute $[7,8]$ if, for all $t \in \mathbb{R}, e^{i t B} A \subset A e^{-i t B}$ (it is shown that this definition is in fact symmetric in $A$ and $B$ ).

The next lemma summarizes some basic facts on strongly commuting (resp., anticommuting) self-adjoint operators.

Lemma 2. Let $A$ and $B$ be self-adjoint operators on $\mathscr{X}$.

(i) A and B strongly commute if and only if, for all $t, s \in \mathbb{R}$, $e^{i t B} e^{i s A}=e^{i s A} e^{i t B}$.

(ii) $A$ and $B$ strongly commute if and only if, for all $t \in \mathbb{R}$, $e^{i t B} A=A e^{i t B}$. (iii) Let $A$ be bounded. Then $A$ and B strongly commute if and only if $A B \subset B A$.

(iv) Let $A$ be bounded. Then $A$ and $B$ strongly anticommute if and only if $A B \subset-B A$.

Proof. Part (i) is well known (e.g., [9, Theorem VIII.13]). Using (i), functional calculus, and strong differential calculus, one can easily prove (ii) and (iii). A proof of (iv) is similar to the proof of (iii).

\section{Description of the Model}

Let $d \geq 2$ be a natural number, and

$$
N_{d}:= \begin{cases}2^{d / 2} & \text { for } d \text { even } \\ 2^{(d+1) / 2} & \text { for } d \text { odd }\end{cases}
$$

Let $\mathscr{K}$ be a separable complex Hilbert space, and

$$
\begin{aligned}
\mathscr{H} & :=L^{2}\left(\mathbb{R}^{d} ; \mathbb{C}^{N_{d}}\right) \otimes \mathscr{K} \cong L^{2}\left(\mathbb{R}^{d} ; \mathbb{C}^{N_{d}} \otimes \mathscr{K}\right) \\
& \cong \int_{\mathbb{R}^{d}}^{\oplus} \mathbb{C}^{N_{d}} \otimes \mathscr{K} d x,
\end{aligned}
$$

where each $\cong$ means the natural Hilbert space isomorphism and $\int_{\mathbb{R}^{d}}^{\oplus} \mathbb{C}^{N_{d}} \otimes \mathscr{K} d x$ denotes the constant fibre direct integral with fiber $\mathbb{C}^{N_{d}} \otimes \mathscr{K}$ (e.g., [10, section XIII.16]).

We denote by $D_{j}$ the generalized partial differential operator in the variable $x_{j}\left(x=\left(x_{1}, \ldots, x_{d}\right) \in \mathbb{R}^{d}\right)$, acting in $L^{2}\left(\mathbb{R}^{d}\right)$. The $d$-dimensional generalized Laplacian

$$
\Delta:=\sum_{j=1}^{d} D_{j}^{2}
$$

on $L^{2}\left(\mathbb{R}^{d}\right)$ is a nonpositive self-adjoint operator. Each linear operator $A$ on $L^{2}\left(\mathbb{R}^{d}\right)$ is extended as the direct sum $\oplus^{N_{d}} A$ on $L^{2}\left(\mathbb{R}^{d} ; \mathbb{C}^{N_{d}}\right)=\oplus^{N_{d}} L^{2}\left(\mathbb{R}^{d}\right)$. For notational simplicity, we denote it by $A$ again.

Every densely defined closable linear operator $T$ on $L^{2}\left(\mathbb{R}^{d}, \mathbb{C}^{N_{d}}\right)$ (resp., $\mathscr{K}$ ) has a tensor product extension $T \otimes$ $I$ (resp., $I \otimes T)$ to $\mathscr{H}$ ( $I$ denotes identity). But we write it $T$ simply if there is no danger of confusion.

We denote by $\mathscr{F}_{\text {s.a. }}$ the set of mappings $\Phi(\cdot)$ from $\mathbb{R}^{d}$ to the set of self-adjoint operators on $\mathbb{C}^{N_{d}} \otimes \mathscr{K}$ such that the mapping: $\mathbb{R}^{d} \ni x \mapsto(\Phi(x)+i)^{-1}$ is measurable. By a general theorem (e.g., [10, Theorem XIII.85(i)]), for each $\Phi(\cdot) \in \mathscr{F}_{\text {s.a. }}$, the direct integral

$$
\Phi:=\int_{\mathbb{R}^{d}}^{\oplus} \Phi(x) d x
$$

is self-adjoint.

Let $\left\{\alpha_{j}\right\}_{j=1}^{d+1}$ be $N_{d} \times N_{d}$ Hermitian matrices satisfying

$$
\left\{\alpha_{j}, \alpha_{k}\right\}=2 \delta_{j k} 1_{N_{d}}, \quad j, k=1, \ldots, d+1 .
$$


Then the free massless Dirac operator on $L^{2}\left(\mathbb{R}^{d} ; \mathbb{C}^{N_{d}}\right)$ is defined by

$$
H_{0}:=-i \sum_{j=1}^{d} \alpha_{j} D_{j}
$$

The operator $H_{0}$ is self-adjoint with $D\left(H_{0}\right):=\bigcap_{j=1}^{d} D\left(D_{j}\right)$ and

$$
H_{0}^{2}=-\Delta \text {. }
$$

To introduce a mass operator, let $M(\cdot) \in \mathscr{F}_{\text {s.a. }}$ such that, for a.e. $x \in \mathbb{R}^{d}, M(x)$ is a bounded operator on $\mathbb{C}^{N_{d}} \otimes \mathscr{K}$, and set

$$
M:=\int_{\mathbb{R}^{d}}^{\oplus} M(x) d x .
$$

We use this self-adjoint operator as an extended mass (variable in the space $\mathbb{R}^{d}$ ) of the quantum particle of our model (a Dirac particle). Note that $M$ is not necessarily bounded.

The Hamiltonian $H$ of our model, a $d$-dimensional version of the GCQS model, is defined as follows:

$$
H:=H_{0}+U,
$$

with

$$
U:=\alpha_{d+1} e^{i \Phi} M
$$

As remarked previously, the mass operator $M$ in $U$ can be variable spatially. This is a point different from the GCQS model.

In this work, we do not intend to discuss essential selfadjointness of $H$ in full generality. In the present paper, we assume the following.

(A.1) $\alpha_{d+1} \Phi \subset-\Phi \alpha_{d+1}$.

(A.2) $\alpha_{d+1} M \subset M \alpha_{d+1}$.

(A.3) $\Phi$ and $M$ strongly commute.

(A.4) The operator $M$ is $(-\Delta)^{1 / 2}$-bounded: $D\left((-\Delta)^{1 / 2}\right) \subset$ $D(M)$ and

$$
\|M \psi\|^{2} \leq a^{2}\left\|(-\Delta)^{1 / 2} \psi\right\|^{2}+b^{2}\|\psi\|^{2}, \quad \forall \psi \in D\left((-\Delta)^{1 / 2}\right)
$$

with constants $0 \leq a<1$ and $b \geq 0$.

Remark 3. In the abstract CQS model [5], the strong commutativity of $M$ and $H_{0}$ as well as the boundedness and the strict positivity of $M$ is assumed. But, in our model, they are not assumed.

Lemma 4. (i) Condition (A.1) holds if and only if $\alpha_{d+1}$ and $\Phi$ strongly anticommute.

(ii) Condition (A.1) is equivalent to the operator equality $\alpha_{d+1} \Phi=-\Phi \alpha_{d+1}$.

(iii) Condition (A.2) holds if and only if $\alpha_{d+1}$ and $M$ strongly commute. (iv) Condition (A.2) is equivalent to the operator equality $\alpha_{d+1} M=M \alpha_{d+1}$.

Proof. (i) This follows from Lemma 2(iv).

(ii) Assume (A.1). Let $\psi \in D\left(\Phi \alpha_{d+1}\right)$. Then $\eta:=\alpha_{d+1} \psi \in$ $D(\Phi)$. Hence, by (A.1), $\alpha_{d+1} \eta \in D(\Phi)$. But, since $\alpha_{d+1}^{2}=I$, we have $\alpha_{d+1} \eta=\psi$. Hence $\psi \in D(\Phi)$. Thereore $D\left(\Phi \alpha_{d+1}\right) \subset D\left(\alpha_{d+1} \Phi\right)$. Thus $D\left(\Phi \alpha_{d+1}\right)=$ $D\left(\alpha_{d+1} \Phi\right)$. Hence the desired operator equality holds.

(iii) This follows from Lemma 2(iii).

(iv) Simillar to the proof of part (ii).

We define

$$
H_{M}:=H_{0}+\alpha_{d+1} M \text {. }
$$

If $M$ is a constant operator $m>0$, then $H_{m}$ represents the free Dirac operator with a constant mass $m$. It is well known (e.g., [6, Theorem 1.1]) that $H_{m}$ is self-adjoint with $D\left(H_{m}\right)=$ $D\left(H_{0}\right)=\cap_{j=1}^{d} D\left(D_{j}\right)$ and bijective with $\left\|H_{m}^{-1}\right\|=1 / m$.

Lemma 5. Assume (A.4). Let $m>0$ be a constant. Then $M H_{m}^{-1}$ is bounded with

$$
\left\|M H_{m}^{-1}\right\| \leq \max \left\{a, \frac{b}{m}\right\} .
$$

Proof. It is well known or easy to see that, for all $\psi \in D\left(H_{m}\right)=$ $D\left(H_{0}\right)$,

$$
\left\|H_{m} \psi\right\|^{2}=\left\|(-\Delta)^{1 / 2} \psi\right\|^{2}+m^{2}\|\psi\|^{2} .
$$

Hence, by (A.4), we have

$$
\|M \psi\|^{2} \leq a^{2}\left\|H_{m} \psi\right\|+\left(b^{2}-a^{2} m^{2}\right)\|\psi\| .
$$

This implies the following: (i) if $a m \leq b$, then $\left\|M H_{m}^{-1}\right\|^{2} \leq$ $a^{2}+\left(b^{2}-a^{2} m^{2}\right) / m^{2}=b^{2} / m^{2}$; (ii) if $a m \geq b$, then $\left\|M H_{m}^{-1}\right\| \leq a$. Thus (17) follows.

Lemma 6. Assume (A.1)-(A.4). Then

(i) $U$ is self-adjoint with $D(U)=D(M)$;

(ii) $H$ is self-adjoint with $D(H)=D\left(H_{0}\right)$ and the subspace

$$
\mathscr{D}_{0}:=C_{0}^{\infty}\left(\mathbb{R}^{d} ; \mathbb{C}^{N_{d}}\right) \widehat{\otimes} \mathscr{K},
$$

( $\widehat{\otimes}$ means algebraic tensor product) is a core of $H$.

Proof. (i) Since $D(U)=D(M)$ and $D(M)$ is dense, $U$ is densely defined. Since $\alpha_{d+1} e^{i \Phi}$ is bounded, it follows that $U^{*}=M e^{-i \Phi} \alpha_{d+1}$. By (A.1) and Lemma 2(iv), we have

$$
e^{-i \Phi} \alpha_{d+1}=\alpha_{d+1} e^{i \Phi}
$$


By (A.2), (A.3), Lemma 2(ii), and Lemma 4(iv), we have

$$
U^{*}=M \alpha_{d+1} e^{i \Phi}=\alpha_{d+1} M e^{i \Phi}=\alpha_{d+1} e^{i \Phi} M=U .
$$

Hence $U$ is self-adjoint.

(ii) By (A.4), we have for all $\psi \in D\left((-\Delta)^{1 / 2}\right)$

$$
\|U \psi\|=\|M \psi\| \leq a\left\|(-\Delta)^{1 / 2} \psi\right\|+b\|\psi\| .
$$

Note that

$$
\left\|(-\Delta)^{1 / 2} \psi\right\|=\left\|H_{0} \psi\right\| .
$$

Hence $\|U \psi\| \leq a\left\|H_{0} \psi\right\|+b\|\psi\|$. Here $0 \leq a<1$. Thus, by the Kato-Rellich theorem (e.g., [11, Theorem X.12]), $H$ is selfadjoint with $D(H)=D\left(H_{0}\right)$ and every core of $H_{0}$ is a core of $H$. It is well known that the subspace $C_{0}^{\infty}\left(\mathbb{R}^{d} ; \mathbb{C}^{N_{d}}\right)$ is a core of $H_{0}$ as a linear operator on $L^{2}\left(\mathbb{R}^{d} ; \mathbb{C}^{N_{d}}\right)$. Hence the subspace $\mathscr{D}_{0}$ defined by (20) is a core of $H_{0}$ as a linear operator on $\mathscr{H}$. Thus it is a core of $H$ too.

Remark 7. One of the other sufficient conditions for $H$ to be essentially self-adjoint is as follows: assume (A.1)-(A.3) and ess $\sup _{|x|<R}\|M(x)\|<\infty$ for all $R>0$. Then $H$ is essentially self-adjoint on $\mathscr{D}_{0}$. The proof is similar to that of [6, Theorem 4.3].

\section{Supersymmetric Aspects}

As is well known, the standard free Dirac operator $-i \sum_{j=1}^{3}$ $\alpha_{j} D_{j}+m \beta$ on $L^{2}\left(\mathbb{R}^{3} ; \mathbb{C}^{4}\right)$ with constant mass $m \geq 0$ and its suitably perturbed ones have supersymmetry; that is, they are, respectively, a supercharge with the grading operator $i \beta \gamma_{5}$ [6, Section 5.5]. From this point of view, it would be interesting to investigate if the Hamiltonian $H$ of the present model has supersymmetry. Indeed, it was shown that the Hamiltonian of the CQS model as well as that of the GCQS model has supersymmetry $[3,4]$. In this section we see that a supersymmetric structure similar to that of the CQS (GCQS) model exists in our model.

In this section, we consider only the case where dis odd. The matrix

$$
\gamma_{5}^{(d)}:=i^{d(d-1) / 2} \alpha_{1} \cdots \alpha_{d}
$$

is self-adjoint with

$$
\left(\gamma_{5}^{(d)}\right)^{2}=1_{N_{d}} .
$$

Since $d$ is odd, we have

$$
\alpha_{j} \gamma_{5}^{(d)}=\gamma_{5}^{(d)} \alpha_{j} \quad(j=1, \ldots, d), \quad\left\{\alpha_{d+1}, \gamma_{5}^{(d)}\right\}=0 .
$$

Let $\xi: \mathbb{R}^{d} \rightarrow \mathfrak{B}(\mathscr{K})$ be Borel measurable such that, for a.e. $x \in \mathbb{R}^{d}, \xi(x)$ is self-adjoint with

$$
\xi(x)^{2}=I .
$$

Then

$$
\|\xi(x)\|=1, \quad \text { a.e. } x \in \mathbb{R}^{d} .
$$

We define $\Gamma(\cdot): \mathbb{R}^{d} \rightarrow \mathfrak{B}\left(\mathbb{C}^{N_{d}} \otimes \mathscr{K}\right)$ by

$$
\Gamma(x):=i \gamma_{5}^{(d)} \alpha_{d+1} \otimes \xi(x), \quad \text { a.e. } x \in \mathbb{R}^{d} .
$$

Then

$$
\Gamma:=\int_{\mathbb{R}^{d}}^{\oplus} \Gamma(x) d x
$$

is self-adjoint with

$$
\Gamma^{2}=I .
$$

Hence $\Gamma$ is a grading operator on $\mathscr{H}$. The following proposition shows that, under some additional condition for $\xi(x), H$ has supersymmetry with respect to $\Gamma$.

Proposition 8. Let d be odd. Assume (A.1)-(A.4). Suppose that $\xi$ is strongly differentiable on $\mathbb{R}^{d}$ with

$$
C_{j}:=\sup _{x \in \mathbb{R}^{d}}\left\|D_{j} \xi(x)\right\|<\infty, \quad j=1, \ldots, d .
$$

Then

$$
\Gamma H \subset-H \Gamma
$$

if and only if

$$
\begin{aligned}
& \sum_{j=1}^{d} \gamma_{5}^{(d)} \alpha_{d+1} \alpha_{j} D_{j} \xi(x) \\
& =i\left(\gamma_{5}^{(d)} \otimes \xi(x) e^{i \Phi(x)} M(x)\right. \\
& \left.\quad-M(x) e^{-i \Phi(x)} \gamma_{5}^{(d)} \otimes \xi(x)\right), \quad \text { a.e. } x \in \mathbb{R}^{d} .
\end{aligned}
$$

In that case, the spectrum $\sigma(H)$ and the point spectrum $\sigma_{p}(H)$ of $H$ are, respectively, symmetric with respect to the origin $0 \in$ $\mathbb{R}$.

Proof. Since the subspace $\mathscr{D}_{0}$ given by (20) is a core of $H$ by Lemma 6(ii), (34) is equivalent to that, for all $\psi \in \mathscr{D}_{0}, \Gamma \psi \in$ $D(H)$ and

$$
\Gamma H \psi=-H \Gamma \psi .
$$

Let $\psi \in \mathscr{D}_{0}$. Then

$$
(\Gamma \psi)(x)=i \gamma_{5}^{(d)} \alpha_{d+1} \otimes \xi(x) \psi(x), \quad \text { a.e. } x \in \mathbb{R}^{d} .
$$

It follows that the $\mathbb{C}^{N_{d}} \otimes \mathscr{K}$-valued function: $x \mapsto(\Gamma \psi)(x)$ is strongly differentiable on $\mathbb{R}^{d}$ with

$$
\begin{array}{r}
D_{j}(\Gamma \psi)(x)=i \gamma_{5}^{(d)} \alpha_{d+1} \otimes\left(D_{j} \xi(x)\right) \psi(x) \\
+i \gamma_{5}^{(d)} \alpha_{d+1} \otimes \xi(x) D_{j} \psi(x), \\
j=1, \ldots, d .
\end{array}
$$

Hence

$$
\left\|D_{j}(\Gamma \psi)(x)\right\|^{2} \leq 2\left(C_{j}^{2}\|\psi(x)\|^{2}+\left\|D_{j} \psi(x)\right\|^{2}\right),
$$


which implies that $D_{j} \Gamma \psi \in \mathscr{H}$ and hence $\Gamma \psi \in D\left(H_{0}\right)=$ $D(H)$. Moreover, we have

$$
\begin{aligned}
&\left(H_{0} \Gamma \psi\right)(x) \\
&=-\gamma_{5}^{(d)} \alpha_{d+1}\left(\sum_{j=1}^{d} \alpha_{j} D_{j} \xi(x)\right) \psi(x)-\left(\Gamma H_{0} \psi\right)(x), \\
&(U \Gamma \psi)(x)=-(\Gamma U \psi)(x) \\
&+i\left(\gamma_{5}^{(d)} \otimes \xi(x) e^{i \Phi(x)} M(x)\right. \\
&\left.-M(x) e^{-i \Phi(x)} \gamma_{5}^{(d)} \otimes \xi(x)\right) \psi(x) .
\end{aligned}
$$

Hence

$$
\begin{aligned}
(H \Gamma \psi)(x)= & -(\Gamma H \psi)(x)-\sum_{j=1}^{d} \gamma_{5}^{(d)} \alpha_{d+1} \alpha_{j} \otimes\left(D_{j} \xi(x)\right) \psi(x) \\
& +i\left(\gamma_{5}^{(d)} \otimes \xi(x) e^{i \Phi(x)} M(x)\right. \\
& \left.-M(x) e^{-i \Phi(x)} \gamma_{5}^{(d)} \otimes \xi(x)\right) \psi(x) .
\end{aligned}
$$

Therefore, $H \Gamma \psi=-\Gamma H \psi$ for all $\psi \in \mathscr{D}_{0}$ if and only if

$$
\begin{aligned}
& \sum_{j=1}^{d} \gamma_{5}^{(d)} \alpha_{d+1} \alpha_{j} \otimes\left(D_{j} \xi(x)\right) \psi(x) \\
& =i\left(\gamma_{5}^{(d)} \otimes \xi(x) e^{i \Phi(x)} M(x)\right. \\
& \left.\quad-M(x) e^{-i \Phi(x)} \gamma_{5}^{(d)} \otimes \xi(x)\right) \psi(x), \quad \forall \psi \in \mathscr{D}_{0} .
\end{aligned}
$$

By the original assumption for $M(\cdot), M(x) \in \mathfrak{B}\left(\mathbb{C}^{N_{d}} \otimes \mathscr{K}\right)$ for a.e. $x \in \mathbb{R}^{d}$. Therefore (42) is equivalent to (35).

By (32) and $\Gamma^{*}=\Gamma$, one easily sees that (34) is in fact equivalent to operator equality $\Gamma^{*} H \Gamma=-H$. Hence $H$ is unitarily equivalent to $-H$. This implies the symmetry of $\sigma(H)$ and $\sigma_{p}(H)$ with respect to the origin.

Remark 9. Proposition 8 gives a generalization of $[4$, Theorem 1] and clarifies a condition for $H$ to have supersymmetry.

It may be difficult in general to show the existence of selfadjoint, unitary solutions $\xi(x)$ to operator equation (35). Here we only note the following fact.

Lemma 10. Let $d$ be odd. Assume (A.1)-(A.3). Suppose that

$$
\gamma_{5}^{(d)} \Phi(x) \subset \Phi(x) \gamma_{5}^{(d)}, \quad \text { a.e. } x \in \mathbb{R}^{d}, j=1, \ldots, d,
$$

$\xi=\xi(x)$ is independent of $x \in \mathbb{R}^{d}$ and

$$
\begin{gathered}
(I \otimes \xi) \Phi(x) \subset-\Phi(x)(I \otimes \xi), \\
\left(\gamma_{5}^{(d)} \otimes \xi\right) M(x) \subset M(x)\left(\gamma_{5}^{(d)} \otimes \xi\right), \quad \text { a.e. } x \in \mathbb{R}^{d} .
\end{gathered}
$$

Then $\xi$ is a solution to (35).
Proof. Since $\xi$ is a constant operator, $D_{j} \xi=0$. By Lemma 2(iii), (43) implies the strong commutativity of $\gamma_{5}^{(d)}$ and $\Phi$. Hence $\gamma_{5}^{(d)} e^{i \Phi(x)}=e^{i \Phi(x)} \gamma_{5}^{(d)}$ for a.e. $x \in \mathbb{R}^{d}$. By (44) and Lemma 2(iv), $(I \otimes \xi) e^{i \Phi(x)}=e^{-i \Phi(x)}(I \otimes \xi)$. We also have (45) and the strong commutativity of $\Phi$ and $M$. Hence

$$
\begin{aligned}
\left(\gamma_{5}^{(d)} \otimes \xi\right) e^{i \Phi(x)} M(x) & =M(x)\left(\gamma_{5}^{(d)} \otimes \xi\right) e^{i \Phi(x)} \\
& =M(x) e^{-i \Phi}\left(\gamma_{5}^{(d)} \otimes \xi\right)
\end{aligned}
$$

Thus (35) holds with both sides being zero.

Additionally we make a remark on the converse of Lemma 10. For this purpose, we need a lemma.

Lemma 11. Let $T_{j}(j=1, \ldots, d)$ be a densely defined closed linear operator on $\mathscr{K}$. Suppose that

$$
\sum_{j=1}^{d} \alpha_{j} \otimes T_{j}=0 \quad \text { on } \cap_{j=1}^{d} D\left(\alpha_{j} \otimes T_{j}\right) .
$$

Then, for all $j=1, \ldots, d, T_{j}=0$ on $\cap_{j=1}^{d} D\left(T_{j}\right)$.

Proof. Equation (47) implies that, for all $u \in \cap_{j=1}^{d} D\left(T_{j}\right)$ and $v \in \mathscr{K}, \sum_{j=1}^{d}\left\langle v, T_{j} u\right\rangle \alpha_{j}=0$. Since $\left\{\alpha_{j}\right\}_{j=1}^{d}$ is linearly independent, it follows that $\left\langle v, T_{j} u\right\rangle=0, j=1, \ldots, d$. Hence $T_{j} u=0, j=1, \ldots, d$.

The following lemma gives a sufficient condition for a solution to (35) to be a constant operator.

Lemma 12. Let $d$ be odd. Assume (A.1)-(A.3). Let $\xi(x)$ be strongly differentiable on $\mathbb{R}^{d}$ with (33) and be a solution to (35). Suppose that (43)-(45) hold. Then $\xi$ is independent of $x \in \mathbb{R}^{d}$.

Proof. As in the proof of Lemma 10, (43)-(45) imply (46). Hence the right-hand side of (35) vanishes, so that $\sum_{j=1}^{d} \gamma_{5}^{(d)} \alpha_{d+1} \alpha_{j} \otimes D_{j} \xi(x)=0$, which implies that $\sum_{j=1}^{d} \alpha_{j} \otimes$ $D_{j} \xi(x)=0$. By Lemma 11, $D_{j} \xi(x)=0, j=1, \ldots, d$, which implies that $\xi$ is independent of $x$.

We have from Proposition 8 and Lemma 10 the following result.

Corollary 13. Let d be odd. Assume (A.1)-(A.4). Suppose that $\xi=\xi(x)$ is independent of $x \in \mathbb{R}^{d}$ and that (43)-(45) hold. Then $H$ has supersymmetry with respect to $\Gamma$.

\section{Vanishing Theorems of the Kernel of $H$}

In supersymmetric quantum mechanics with a supercharge $Q$, a nonzero vector in $\operatorname{ker} Q$ is called a supersymmetric states. If the kernel of $Q$ vanishes, that is, $\operatorname{ker} Q=\{0\}$, then the supersymmetry is said to be spontaneously broken. It turns out that, in supersymmetric quantum mechanics, it is importanat to investigate ker $Q$. Thus we are led to consider ker $H$ in view of Proposition 8. This would be interesting even if $H$ does not 
have supersymmetry (note that $H$ does not necessarily have supersymmetry).

To investigate ker $H$, we also need an additional condition.

(A.5) (i) For each $f \in \mathbb{C}^{N_{d}} \otimes \mathscr{K}$, the function: $x \mapsto M(x) f$ is strongly differentiable on $\mathbb{R}^{d}$ and, for all $x \in \mathbb{R}^{d}$, $M(x)$ commutes with $\alpha_{j}(j=1, \ldots, d)$. (ii) There exists a constant $\mu_{0}>0$ such that

$M(x)^{2}-i \sum_{j=1}^{d} \alpha_{j} \alpha_{d+1} D_{j} M(x) \geq \mu_{0}^{2}, \quad \forall x \in \mathbb{R}^{d}$

as an operator inequality on $\mathbb{C}^{N_{d}} \otimes \mathscr{K}$ (note that, by the principle of uniform boundedness, the strong partial derivative $D_{j} M(x)$ is a bounded operator on $\mathbb{C}^{N_{d}} \otimes \mathscr{K}$ for each $x \in \mathbb{R}^{d}$ and hence, under (A.2) and condition (A.5)(i), the operator $M(x)^{2}-i \sum_{j=1}^{d} \alpha_{j} \alpha_{d+1} D_{j} M(x)$ on $\mathbb{C}^{N_{d}} \otimes \mathscr{K}$ is a bounded self-adjoint operator).

For a linear operator $L$ on a Hilbert space, we denote the resolvent set of $L$ by $\rho(L)$.

Lemma 14. Assume (A.2), (A.4), and (A.5). Then $H_{M}$ defined by (16) is self-adjoint with $D\left(H_{M}\right)=D\left(H_{0}\right)$ and

$$
\left\|H_{0} \psi\right\|^{2}+\mu_{0}^{2}\|\psi\|^{2} \leq\left\|H_{M} \psi\right\|^{2}, \quad \psi \in D\left(H_{0}\right) .
$$

In particular, $0 \in \rho\left(H_{M}\right)$ with operator-norm bound

$$
\left\|H_{M}^{-1}\right\| \leq \frac{1}{\mu_{0}}
$$

and $H_{0} H_{M}^{-1}$ is bounded with

$$
\left\|H_{0} H_{M}^{-1}\right\| \leq 1
$$

Moreover, $M H_{M}^{-1}$ is bounded with

$$
\left\|M H_{M}^{-1}\right\| \leq a+\frac{b}{\mu_{0}} .
$$

Proof. The self-adjointness of $H_{M}$ follows from that of $H$ with $\Phi=0$. For all $\psi \in \mathscr{D}_{0}$, using the anticommutativity of $\alpha_{j}$ with $\alpha_{d+1}$ and the commutativity of $M(x)$ with $\alpha_{d+1}$ and $\alpha_{j}$ $(j=1, \ldots, d)$, we have

$$
\begin{aligned}
& \left\|H_{M} \psi\right\|^{2} \\
& =\left\|H_{0} \psi\right\|^{2}+\|M \psi\|^{2} \\
& \quad+\sum_{j=1}^{d}\left\langle\psi,\left(-i D_{j} M\right) \alpha_{j} \alpha_{d+1} \psi\right\rangle \\
& \geq \|\left. H_{0} \psi\right|^{2}
\end{aligned}
$$

$$
\begin{aligned}
& +\int_{\mathbb{R}^{d}}\left\langle\psi(x),\left(M(x)^{2}\right.\right. \\
& \left.\left.\quad-i \sum_{j=1}^{d} \alpha_{j} \alpha_{d+1} D_{j} M(x)\right) \psi(x)\right\rangle d x \\
& \geq\left\|H_{0} \psi\right\|^{2}+\mu_{0}^{2}\|\psi\|^{2} .
\end{aligned}
$$

Hence (49) holds for all $\psi \in \mathscr{D}_{0}$. Since $\mathscr{D}_{0}$ is a core of $H_{M}$, this inequality extends to all $\psi \in D\left(H_{0}\right)$. In particular, we have

$$
\mu_{0}\|\psi\| \leq\left\|H_{M} \psi\right\|, \quad \psi \in D\left(H_{0}\right) .
$$

This implies that the self-adjoint operator $H_{M}$ is bijective with (50).

Inequality (49) implies also that, for all $\psi \in D\left(H_{0}\right)$, $\left\|H_{0} \psi\right\| \leq\left\|H_{M} \psi\right\|$. Hence $H_{0} H_{M}^{-1}$ is bounded with (51).

By (A.4) and $\left\|(-\Delta)^{1 / 2} \psi\right\|=\left\|H_{0} \psi\right\|$ for all $\psi \in D$ $\left((-\Delta)^{1 / 2}\right)=D\left(H_{0}\right)$, we have $\|M \psi\| \leq a\left\|H_{0} \psi\right\|+b\|\psi\|$. Hence, for all $\phi \in \mathscr{H}$,

$$
\begin{aligned}
\left\|M H_{M}^{-1} \phi\right\| & \leq a\left\|H_{0} H_{M}^{-1} \phi\right\|+b\left\|H_{M}^{-1} \phi\right\| \\
& \leq\left(a\left\|H_{0} H_{M}^{-1}\right\|+b\left\|H_{M}^{-1}\right\|\right)\|\phi\| \\
& \leq\left(a+\frac{b}{\mu_{0}}\right)\|\phi\| .
\end{aligned}
$$

Thus (52) holds.

Lemma 15. Let $A$ be a self-adjoint operator on a complex Hilbert space $\mathscr{X}$. Then

$$
\left\|e^{i A}-1\right\|=2\left\|\sin \frac{A}{2}\right\| .
$$

Proof. By the functional calculus, one has $e^{i A}-1=$ $2 i e^{i A / 2} \sin (A / 2)$. Hence $\left\|e^{i A}-1\right\|=2\left\|e^{i A / 2} \sin (A / 2)\right\|$. Since $e^{i A / 2}$ is unitary, one has $\left\|e^{i A / 2} \sin (A / 2)\right\|=\|\sin (A / 2)\|$. Thus (56) holds.

Theorem 16. Assume (A.1)-(A.5) and

$$
\text { ess } \sup _{x \in \mathbb{R}^{d}}\left\|\sin \frac{\Phi(x)}{2}\right\|<\frac{1}{2\left(a+b \mu_{0}^{-1}\right)} .
$$

Then $\operatorname{ker} H=\{0\}$ and $0 \in \rho(H)$.

Moreover, the constant

$$
\gamma(H):=\inf _{\psi \in D(H),\|\psi\|=1}\|H \psi\|
$$

is strictly positive, $\gamma(H) \in \sigma(H)$ or $-\gamma(H) \in \sigma(H)$, and

$$
\sigma(H) \subset(-\infty,-\gamma(H)] \cup[\gamma(H), \infty) .
$$

Proof. The operator $H$ is written as

$$
H=H_{M}+\alpha_{d+1}\left(e^{i \Phi}-1\right) M=K H_{M}
$$


with

$$
K:=I+\alpha_{d+1}\left(e^{i \Phi}-1\right) M H_{M}^{-1} .
$$

By applying Lemma 15 with $A=\Phi(x)$, we have

$$
\left\|e^{i \Phi(x)}-1\right\|=2\left\|\sin \frac{\Phi(x)}{2}\right\| .
$$

Therefore, for all $\psi \in \mathscr{H}$,

$$
\begin{aligned}
& \left\|\alpha_{d+1}\left(e^{i \Phi}-1\right) M H_{M}^{-1} \psi\right\| \\
& \leq 2 \operatorname{ess} \sup _{x \in \mathbb{R}^{d}}\left\|\sin \left(\frac{\Phi(x)}{2}\right)\right\|\left\|M H_{M}^{-1}\right\|\|\psi\| .
\end{aligned}
$$

By this estimate and (52), we obtain

$$
\begin{aligned}
& \left\|\alpha_{d+1}\left(e^{i \Phi}-1\right) M H_{M}^{-1}\right\| \\
& \quad \leq 2\left(\operatorname{ess~sup~}_{x \in \mathbb{R}^{d}}\left\|\sin \left(\frac{\Phi(x)}{2}\right)\right\|\right)\left(a+b \mu_{0}^{-1}\right) .
\end{aligned}
$$

Hence, by (57), we obtain $\left\|\alpha_{d+1}\left(e^{i \Phi}-1\right) M H_{M}^{-1}\right\|<1$. This implies that $K$ is bijective with bounded inverse $K^{-1}$. Thus $H$ is bijective with $H^{-1}=H_{M}^{-1} K^{-1}$ being bounded. Hence ker $H=\{0\}$ and $0 \in \rho(H)$.

We set $b_{H}:=\left\|H^{-1}\right\|$. If $|\lambda|<1 / b_{H}$, then $\lambda$ is in $\rho(H)$. Therefore

$$
\sigma(H) \subset\left(-\infty,-b_{H}^{-1}\right] \cup\left[b_{H}^{-1}, \infty\right) .
$$

It is obvious that, for all $\psi \in D(H)$ with $\|\psi\|=1,1 \leq$ $b_{H}\|H \psi\|$. This implies that $b_{H} \gamma(H) \geq 1$. On the other hand, we have from (58) $\|\psi\| \geq \gamma(H)\left\|H^{-1} \psi\right\|$, for all $\psi \in \mathscr{H}$. Hence $b_{H} \gamma(H) \leq 1$. Therefore $b_{H}^{-1}=\gamma(H)$. Thus (59) holds and $\gamma(H)>0$. Since $b_{H} \in \sigma\left(H^{-1}\right)$ or $-b_{H} \in \sigma\left(H^{-1}\right)$, it follows that $\gamma(H)=b_{H}^{-1} \in \sigma(H)$ or $-\gamma(H) \in \sigma(H)$.

Remark 17. Under the same assumption as in Theorem 16, $H$ is Fredholm (the proof is easy).

We next consider a perturbation of $\Phi(\cdot)$. Let $\eta(\cdot) \epsilon$ $\mathscr{F}_{\text {s.a. }}$ such that, for a.e. $x \in \mathbb{R}^{d}, \eta(x)$ is bounded and strongly commutes with $\Phi(x)$ and $M(x)$. Then, for a.e. $x \in \mathbb{R}^{d}$,

$$
\Phi_{\eta}(x):=\Phi(x)+\eta(x)
$$

is self-adjoint on $\mathbb{C}^{N_{d}} \otimes \mathscr{K}$ and

$$
\Phi_{\eta}:=\int_{\mathbb{R}^{d}}^{\oplus} \Phi_{\eta}(x) d x
$$

is a self-adjoint operator on $\mathscr{H}$.

The quantity

$$
\kappa(H):=\sup _{\psi \in D(H),\|\psi\|=1} \frac{\|M \psi\|}{\|H \psi\|},
$$

may be infinite. But we have the following.
Lemma 18. Under the assumption of Theorem $16,0<\kappa(H)<$ $\infty$.

Proof. Since $H$ is closed with $D(H)=D\left(H_{0}\right) \cap D(U)(=$ $\left.D\left(H_{0}\right)\right)$ and $\|U \psi\|=\|M \psi\|, \psi \in D(H)$, it follows from the closed graph theorem that there exists a constant $c>0$ such that

$$
\|M \psi\| \leq c(\|H \psi\|+\|\psi\|), \quad \psi \in D(H) .
$$

Let $\psi \in D(H)$ with $\|\psi\|=1$. Then, by Theorem 16, we have $\|H \psi\| \geq \gamma(H)>0$. Hence

$$
\frac{\|M \psi\|}{\|H \psi\|} \leq c+\frac{c}{\gamma(H)} .
$$

Therefore $\kappa(H) \leq c+c / \gamma(H)<\infty$. If $\kappa(H)=0$, then $\|M \psi\|=0$ for all $\psi \in D(M)=D\left(H_{0}\right)$. Hence $M=0$. But this contradicts condition (A.5).

Theorem 19. Assume (A.1)-(A.5) and (57). Suppose that

$$
\underset{x \in \mathbb{R}^{d}}{\operatorname{ess} \sup }\left\|\sin \frac{\eta(x)}{2}\right\|<\frac{1}{2 \kappa(H)} .
$$

Let

$$
H_{\eta}:=H_{0}+\alpha_{d+1} e^{i \Phi_{\eta}} M .
$$

Then ker $H_{\eta}=\{0\}$ and $0 \in \rho\left(H_{\eta}\right)$. Moreover, the last statement on $\gamma(H)$ and $\sigma(H)$ in Theorem 16 holds with $H$ being replaced by $H_{\eta}$

Proof. We write

$$
H_{\eta}=H+W, \quad W:=\alpha_{d+1}\left(e^{i \Phi_{\eta}}-e^{i \Phi}\right) M .
$$

By the strong commutativity of $\Phi(x)$ and $\eta(x)$, we have for a.e. $x \in \mathbb{R}^{d}$

$$
\begin{aligned}
e^{i \Phi_{\eta(x)}}-e^{i \Phi(x)} & =e^{i \Phi(x)}\left(e^{i \eta(x)}-1\right) \\
& =2 i e^{i \Phi(x)} e^{i \eta(x) / 2} \sin \left(\frac{\eta(x)}{2}\right) .
\end{aligned}
$$

Hence, for all $\psi \in D\left(H_{0}\right)$

$$
\|W \psi\| \leq \underset{x \in \mathbb{R}^{d}}{2 \operatorname{ess} \sup }\left\|\sin \frac{\eta(x)}{2}\right\|\|M \psi\| .
$$

We have $\|M \psi\| \leq \kappa(H)\|H \psi\|$. Hence

$$
\|W \psi\| \leq C_{\eta}\|H \psi\|
$$

with

$$
C_{\eta}:=2 \kappa(H) \operatorname{ess} \sup _{x \in \mathbb{R}^{d}}\left\|\sin \frac{\eta(x)}{2}\right\| .
$$

Hence $W$ is $H$-bounded. By Remark 17, $H$ is Fredholm. Condition (71) is equivalent to $C_{\eta}<1$. Hence, by a stability theorem (e.g., [12, Chapter IV, Theorem 5.22]), $H_{\eta}$ is Fredholm and $\operatorname{dim} \operatorname{ker} H_{\eta} \leq \operatorname{dim} \operatorname{ker} H=0$. Therefore ker $H_{\eta}=\{0\}$. It follows from this fact and the self-adjointness of $H_{\eta}$ that $\operatorname{Ran}\left(H_{\eta}\right)=\mathscr{H}$. Hence $0 \in \rho\left(H_{\eta}\right)$. Then the last statement of the present theorem can be proved in the same way as in the proof of the corresponding part in Theorem 16. 


\section{Unitary Equivalence to a Gauge Theoretic Dirac Operator and a Vanishing Theorem for $\operatorname{ker} H$}

In the papers $[3,4]$, it was shown that, under a suitable condition, the Hamiltonian of the CQS (GCQS) model is unitarily transformed to a Dirac operator which is simpler in a sense. In this section, we show that those structures are unified into a simple general structure.

We introduce a class of $\Phi(\cdot)$ :

$$
\begin{gathered}
\mathscr{F}:=\left\{\Phi(\cdot) \in \mathscr{F}_{\text {s.a. }} \mid e^{ \pm i \Phi(\cdot) / 2}\right. \text { is strongly differentiable } \\
\text { and } \left.\sup _{x \in \mathbb{R}^{d}}\left\|E_{j}(x)\right\|<\infty, j=1, \ldots, d\right\},
\end{gathered}
$$

where

$$
E_{j}(x):=D_{j} e^{-i \Phi(x) / 2}
$$

denotes the strong partial derivative of $e^{-i \Phi(x) / 2}$ in $x_{j}$. For $\Phi(\cdot) \in \mathscr{F}$, one can define a bounded linear operator

$$
A_{j}:=i \int_{\mathbb{R}^{d}}^{\oplus} e^{i \Phi(x) / 2} E_{j}(x) d x
$$

on $\mathscr{H}$.

Remark 20. If $\Phi(\cdot) \in \mathscr{F}$ sucht that $\Phi(x)$ and $\Phi\left(x^{\prime}\right)$ commute for a.e. $x, x^{\prime} \in \mathbb{R}^{d}$, then $E_{j}(x)=-i e^{-i \Phi(x) / 2} D_{j} \Phi(x) / 2$ and hence

$$
A_{j}=\frac{1}{2} \int_{\mathbb{R}^{d}}^{\oplus} D_{j} \Phi(x) d x .
$$

Lemma 21. For each $j=1, \ldots, d, A_{j}$ is a bounded self-adjoint operator on $\mathscr{H}$.

Proof. Since ess $\sup _{x \in \mathbb{R}^{d}}\left\|e^{i \Phi(x) / 2} E_{j}(x)\right\|=\quad$ ess $\sup _{x \in \mathbb{R}^{d}}$ $\left\|E_{j}(x)\right\|<\infty, A_{j}$ is bounded. We have

$$
A_{j}^{*}=\frac{(-i)}{2} \int_{\mathbb{R}^{d}}^{\oplus}\left(D_{j} e^{i \Phi(x) / 2}\right) e^{-i \Phi(x) / 2} d x .
$$

Differentiating the identity $e^{i \Phi(x) / 2} e^{-i \Phi(x) / 2}=I$ in $x_{j}$, we have

$$
e^{i \Phi(x) / 2} E_{j}(x)=-\left(D_{j} e^{\Phi(x) / 2}\right) e^{-i \Phi(x) / 2} .
$$

Hence $A_{j}^{*}=A_{j}$.

For $\Phi(\cdot) \in \mathscr{F}$, we define an operator:

$$
H^{\prime}:=\sum_{j=1}^{d} \alpha_{j}\left(-i D_{j}-A_{j}\right)+\alpha_{d+1} M=H_{M}-\sum_{j=1}^{d} \alpha_{j} A_{j} .
$$

Lemma 22. Assume (A.4). Let $\Phi(\cdot) \in \mathscr{F}$. Suppose that

$$
\alpha_{j} \Phi \subset \Phi \alpha_{j}, \quad j=1, \ldots, d .
$$

Then $H^{\prime}$ is self-adjoint and every core of $H_{0}$ is a core of $H^{\prime}$.
Proof. Under condition (A.4), $H_{M}$ is self-adjoint. By (85), we have $\alpha_{j} A_{j}=A_{j} \alpha_{j}(j=1, \ldots, d)$. Hence, by Lemma 21, $-\sum_{j=1}^{d} \alpha_{j} A_{j}$ is a bounded self-adjoint operator. Hence the Kato-Rellich theorem yields the desired result.

We note that, if one regards $\mathbf{A}:=\left(A_{1}, \ldots, A_{d}\right)$ as a (noncommutative) gauge potential, then $H^{\prime}$ is a gauge theoretic Dirac operator with gauge potential A.

Let

$$
U:=e^{i \Phi / 2}
$$

which is unitary. The following theorem shows that, under a suitable condition, $H$ is unitarily equivalent to a gauge theoretic Dirac operator $H^{\prime}$.

Theorem 23. Assume (A.1)-(A.4) and (85). Let $\Phi(\cdot) \in \mathscr{F}$. Then

$$
U H U^{-1}=H^{\prime}
$$

Proof. We have

$$
\begin{aligned}
U H U^{-1}= & -i \sum_{j=1}^{d}\left(U \alpha_{j} U^{-1}\right) U D_{j} U^{-1} \\
& +U \alpha_{d+1} e^{i \Phi} U^{-1}\left(U M U^{-1}\right) .
\end{aligned}
$$

By (85) and Lemma $2, U \alpha_{j} U^{-1}=\alpha_{j}$. By (A.3) and Lemma 2, $U M U^{-1}=M$. By (A.1) and Lemma 2(iv), $U \alpha_{d+1} e^{i \Phi} U^{-1}=$ $\alpha_{d+1} e^{-i \Phi / 2} e^{i \Phi} e^{i \Phi / 2}=\alpha_{d+1}$. Moreover,

$$
U D_{j} U^{-1}=D_{j}-i A_{j}
$$

Hence (87) holds.

The following theorem gives another sufficient condition for ker $H$ to be trivial.

Theorem 24. Assume (A.1)-(A.4) and (85). Let $\Phi(\cdot) \in \mathscr{F}$ and

$$
\sum_{j=1}^{d} \text { ess } \sup _{x \in \mathbb{R}^{d}}\left\|E_{j}(x)\right\|<\mu_{0}
$$

Then $\operatorname{ker} H=\{0\}$ and $0 \in \rho(H)$.

Proof. We write $H^{\prime}=H_{M}+X$ with $X:=-\sum_{j=1}^{d} \alpha_{j} A_{j}$. Then

$$
\|X\| \leq \sum_{j=1}^{d}\left\|\alpha_{j} A_{j}\right\| \leq \sum_{j=1}^{d} \text { ess } \sup _{x \in \mathbb{R}^{d}}\left\|E_{j}(x)\right\| .
$$

By this estimate and (90), $\left\|X H_{M}^{-1}\right\|<1$. Hence $H^{\prime}$ is bijective and $0 \in \rho\left(H^{\prime}\right)$. In particular, ker $H^{\prime}=\{0\}$. On the other hand, (87) implies that $\rho\left(H^{\prime}\right)=\rho(H)$ and ker $H=U^{-1}$ ker $H^{\prime}$. Thus $0 \in \rho(H)$ and $\operatorname{ker} H=\{0\}$. 


\section{Essential Spectrum of $H$}

In this section, we consider the essential spectrum of $H$. For a self-adjoint operator $S$ on a Hilbert space, we denote by $\sigma_{\text {ess }}(S)$ the essential spectrum of $S$.

Lemma 25. Let $\operatorname{dim} \mathscr{K}<\infty$ and $m>0$ be a constant. Let $V(\cdot): \mathbb{R}^{d} \rightarrow \mathfrak{B}\left(\mathbb{C}^{N_{d}} \otimes \mathscr{K}\right)$ be Borel measurable satisfying the following conditions.

(i) The operator $V:=\int_{\mathbb{R}^{d}}^{\oplus} V(x) d x$ is relatively bounded with respect to $H_{0}$.

(ii) $\lim _{|x| \rightarrow \infty}\|V(x)\|=0$.

(iii) The operator $H_{m}+V$ on $\mathscr{H}$ is self-adjoint.

Then

$$
\sigma_{e s s}\left(H_{m}+V\right)=(-\infty,-m] \cup[m, \infty) .
$$

Proof. For each $R>0$, we denote by $\chi_{R}$ the characteristic function of the set $\left\{x \in \mathbb{R}^{d}|| x \mid<R\right\}$. As in the case of the 3dimensional free Dirac operator (cf. [6, Lemma 4.6]), one can show that $\left|H_{m}\right|^{-k} \chi_{R}$ is compact for all $k>0$ as an operator on $L^{2}\left(\mathbb{R}^{d} ; \mathbb{C}^{N_{d}}\right)$. Since $\operatorname{dim} \mathscr{K}<\infty$, it follows that $\left|H_{m}\right|^{-k} \chi_{R}$ is compact as an operator on $\mathscr{H}$. Hence, for all $z \in \mathbb{C} \backslash \mathbb{R},\left(H_{m}-\right.$ $z)^{-1} \chi_{R}=\left(\left(H_{m}-z\right)^{-1}\left|H_{m}\right|\right)\left|H_{m}\right|^{-1} \chi_{R}$ is compact. Since $H_{m}+V$ is self-adjoint with $D\left(H_{m}+V\right)=D\left(H_{m}\right)=D\left(H_{0}\right)$ and $V$ is closed, it follows from the closed graph theorem that $V\left(\mathrm{H}_{m}+\right.$ $V-z)^{-1}$ is bounded (this can be shown by direct estimates too). Therefore we have

$$
\begin{aligned}
& \left(H_{m}+V-z\right)^{-1}-\left(H_{m}-z\right)^{-1} \\
& \quad=-\left(H_{m}-z\right)^{-1} V\left(H_{m}+V-z\right)^{-1}=-W_{R}-X_{R},
\end{aligned}
$$

where $W_{R}:=\left(H_{m}-z\right)^{-1} \chi_{R}\left[V\left(H_{m}+V-z\right)^{-1}\right], X_{R}=\left(H_{m}-\right.$ $z)^{-1}\left(1-\chi_{R}\right) V\left(H_{m}+V-z\right)^{-1}$. By the fact mentioned previously, $W_{R}$ is compact. We have

$$
\left\|X_{R}\right\| \leq \frac{1}{|\operatorname{Im} z|^{2}}\left\|\left(1-\chi_{R}\right) V\right\| .
$$

By condition (ii), for every $\varepsilon>0$, there exists a constant $R>0$ such that, for all a.e. $x \in \mathbb{R}^{d}$ with $|x| \geq R,\|V(x)\|<\varepsilon$, that is, ess $\sup _{|x| \geq R}\|V(x)\| \leq \varepsilon$, which implies that $\left\|\left(1-\chi_{R}\right) V\right\| \leq \varepsilon$. Hence $\lim _{R \rightarrow \infty}\left\|X_{R}\right\|=0$. Therefore $\left(H_{m}+V-z\right)^{-1}-\left(H_{m}-\right.$ $z)^{-1}$ is compact. Hence, by Weyl's essential spectrum theorem (e.g., [10, Theorem XIII.14]), $\sigma_{\text {ess }}\left(H_{m}+V\right)=\sigma_{\text {ess }}\left(H_{m}\right)$. On the other hand, as in the case of the 3-dimensional free Dirac operator [6, Theorem 1.1], one can show that $\sigma\left(H_{m}\right)=$ $\sigma_{\text {ess }}\left(H_{m}\right)=(-\infty,-m] \cup[m, \infty)$. Thus (92) holds.

Theorem 26. Let $\operatorname{dim} \mathscr{K}<\infty$. Assume (A.1)-(A.4). Suppose that there exists a constant $m \in \mathbb{R}$ satisfying

$$
\begin{aligned}
& \lim _{|x| \rightarrow \infty}\|M(x)-m\|=0, \\
& \lim _{|x| \rightarrow \infty}\left\|\sin \frac{\Phi(x)}{2}\right\|=0 .
\end{aligned}
$$

Then

$$
\sigma_{\text {ess }}(H)=(-\infty,-m] \cup[m, \infty) .
$$

Proof. We write

$$
H=H_{m}+V_{1}+V_{2}
$$

with

$$
V_{1}:=\alpha_{d+1}(M-m), \quad V_{2}:=\alpha_{d+1}\left(e^{i \Phi}-1\right) M .
$$

It is obvious that $V_{1}$ and $V_{2}$ are relatively bounded with respect to $H_{0}$ and

$$
\lim _{|x| \rightarrow \infty}\left\|V_{1}(x)\right\|=\lim _{|x| \rightarrow \infty}\|M(x)-m\|=0 .
$$

As for $V_{2}$, we have

$$
\left\|V_{2}(x)\right\| \leq\|M(x)\|\left\|e^{i \Phi(x)}-1\right\| \leq 2\|M(x)\|\left\|\sin \frac{\Phi(x)}{2}\right\| .
$$

Hence, by (95) and (96), we have $\lim _{|x| \rightarrow \infty}\left\|V_{2}(X)\right\|=0$. Therefore $\lim _{|x| \rightarrow \infty}\left\|V_{1}(x)+V_{2}(x)\right\|=0$. Thus we can apply Lemma 25 to obtain (97).

If $\Phi(\cdot)$ is in the class $\mathscr{F}$ introduced in Section 6, then we can obtain a sufficient condition for (97) to hold.

Theorem 27. Let $\operatorname{dim} \mathscr{K}<\infty$. Assume (A.1)-(A.4) (85) and (95). Let $\Phi(\cdot) \in \mathscr{F}$. Suppose that

$$
\lim _{|x| \rightarrow \infty}\left\|E_{j}(x)\right\|=0 .
$$

Then (97) holds.

Proof. By (87), we have $\sigma_{\text {ess }}(H)=\sigma_{\text {ess }}\left(H^{\prime}\right)$. Hence we need only to prove

$$
\sigma_{\text {ess }}\left(H^{\prime}\right)=(-\infty,-m] \cup[m, \infty) .
$$

We write

$$
H^{\prime}=H_{m}+\alpha_{d+1}(M-m)-\sum_{j=1}^{d} \alpha_{j} A_{j} .
$$

We have $\lim _{|x| \rightarrow \infty}\left\|\alpha_{d+1}(M(x)-m)\right\|=0$. Moreover, $\left\|\alpha_{j} A_{j}(x)\right\| \leq\left\|E_{j}(x)\right\|$. Hence $\lim _{|x| \rightarrow \infty}\left\|-\sum_{j=1}^{d} \alpha_{j} A_{j}(x)\right\|=0$. Thus we can apply Lemma 25 to obtain (103).

\section{Bounds on the Number of Discrete Eigenvalues}

In this section, in view of Theorem 26, we consider the number of eigenvalues of $H$ in the interval $(-m, m)$ and establish upper bounds on it. This aspect has been considered in the CQS model [3] as well as the GCQS model [4]. In this paper, we take another method, which is an extension of the method used in [13] where the number of eigenvalues of the three-dimensional Dirac operator $H_{m}+V$ with a scalar potential $V: \mathbb{R}^{3} \rightarrow \mathbb{R}$ in $(-m, m)$ is considered. This extension is not difficult. But, for the sake of completeness, we present some details of it. One easily notes that the problem under consideration can be studied in a more general frame work as in Lemma 25. Hence we first discuss the general case. 
8.1. A General Case. Let $V$ be as in Lemma 25 and

$$
H(V):=H_{m}+V
$$

Then, by (92), an eigenvalue of $H(V)$ in $(-m, m)$ (if it exists) is an isolated eigenvalue of $H(V)$ with finite multiplicity. For each $\lambda \in\left(0, m^{2}\right)$, we denote by $N(\lambda, V)$ the number of eigenvalues in the interval $\left(-\sqrt{m^{2}-\lambda}, \sqrt{m^{2}-\lambda}\right)$.

We first note an elementary fact:

Theorem 28. Suppose that the assumption of Lemma 25 holds and that $\|V(x)\| \leq \lambda / 4 m$ for a.e. $x \in \mathbb{R}^{d}$. Then $N(\lambda, V)=0$.

Proof. Suppose that $N(\lambda, V) \geq 1$. Then, it follows from the definition of $N:=N(\lambda, V)$ that there exists an $N$-dimensional subspace $E$ of $\mathscr{H}$ such that

$$
\|H(V) \psi\| \leq \sqrt{m^{2}-\lambda}\|\psi\|, \quad \forall \psi \in E
$$

Hence

$$
\begin{aligned}
\left\|H_{m} \psi\right\| & \leq\|H(V) \psi\|+\|V \psi\| \leq\left(\sqrt{m^{2}-\lambda}+\frac{\lambda}{4 m}\right)\|\psi\| \\
& \leq \sqrt{m^{2}-\frac{\lambda}{2}}\|\psi\| .
\end{aligned}
$$

Hence $\left\|H_{m} \psi\right\|^{2} \leq\left(m^{2}-(\lambda / 2)\right)\|\psi\|^{2}$, which is equivalent to $\left\|(-\Delta+(\lambda / 2))^{1 / 2} \psi\right\|^{2} \leq 0$. This implies that $\psi=0$. But this is a contradiction.

In view of Theorem 28, we define, for each $\lambda>0, V_{\lambda}$ : $\mathbb{R}^{d} \rightarrow \mathfrak{B}\left(\mathbb{C}^{N_{d}} \otimes \mathscr{K}\right)$ by

$$
V_{\lambda}(x):= \begin{cases}V(x) & \text { if }\|V(x)\|>\frac{\lambda}{4 m} \\ 0 & \text { otherwise. }\end{cases}
$$

For each $\lambda>0$, the operator

$$
R_{\lambda}:=\left(-\Delta+\frac{\lambda}{2}\right)^{-1 / 2}
$$

is a bounded self-adjoint operator. Since $V$ is $H_{0}$-bounded, where $H_{0}$ is defined by $(10)$ and $\left(H_{0}+i\right) R_{\lambda}$ is bounded, it follows that $V R_{\lambda}$ and $V_{\lambda} R_{\lambda}$ are bounded operators on $\mathscr{H}$. Also $H_{0} R_{\lambda}$ is bounded with $\left\|H_{0} R_{\lambda}\right\| \leq 1$. Hence the following operators $T_{\lambda j}(j=1,2,3,5)$ are in $\mathfrak{B}(\mathscr{H})$ :

$$
\begin{aligned}
& T_{\lambda 1}:=\left(H_{0} R_{\lambda}\right)^{*} V_{\lambda} R_{\lambda}, \\
& T_{\lambda 2}:=\left(V_{\lambda} R_{\lambda}\right)^{*} H_{0} R_{\lambda}, \\
& T_{\lambda 3}:=m \alpha_{d+1} R_{\lambda} V_{\lambda} R_{\lambda}, \\
& T_{\lambda 4}:=m R_{\lambda} V_{\lambda} R_{\lambda} \alpha_{d+1}, \\
& T_{\lambda 5}:=\left(V_{\lambda} R_{\lambda}\right)^{*} V_{\lambda} R_{\lambda} .
\end{aligned}
$$

We set

$$
v(x):=\|V(x)\|, \quad v_{\lambda}(x):=\left\|V_{\lambda}(x)\right\|, \quad \text { a.e. } x \in \mathbb{R}^{d} .
$$

For a compact operator $A$ on a Hilbert space, we denote the nonincreasing sequence of the singular values of $A$ (repeated with multiplicity) by $\mu_{n}(A)(n \in \mathbb{N})$. For $f \in$ $L^{p}\left(\mathbb{R}^{d}\right)$, we set $\|f\|_{L^{p}}:=\left(\int_{\mathbb{R}^{d}}|f(x)|^{p} d x\right)^{1 / p}$.

Lemma 29. Let $d \geq 3$ and suppose that the assumption of Lemma 25 holds and $v \in L^{d}\left(\mathbb{R}^{d}\right) \cap L^{d / 2}\left(\mathbb{R}^{d}\right)$. Then, for all $j=$ $1,2,3,4,5, T_{\lambda j}$ is compact. Moreover, there exists a constant $C>0$ independent of $V$ and $\lambda>0$ such that, for all $n \in \mathbb{N}$,

$$
\begin{gathered}
\mu_{n}\left(T_{\lambda j}\right) \leq C\left\|v_{\lambda}\right\|_{L^{d}} n^{-1 / d} \quad(j=1,2), \\
\mu_{n}\left(T_{\lambda j}\right) \leq C\left\|v_{\lambda}^{1 / 2}\right\|_{L^{d}}^{2} n^{-2 / d} \quad(j=3,4), \\
\mu_{n}\left(T_{\lambda 5}\right) \leq C\left\|v_{\lambda}\right\|_{L^{d}}^{2} n^{-2 / d} .
\end{gathered}
$$

Proof. By the weak Hausdorff-Young inequality (e.g., [11, page $32]$ ) and the condition $d \geq 3$, one can easily see that the Fourier transform $g_{\lambda}$ of the function: $\mathbb{R}^{d} \ni k \mapsto\left(k^{2}+\lambda / 2\right)^{-1 / 2}$ is in $L_{\mathrm{w}}^{p^{\prime}}\left(\mathbb{R}^{d}\right)$ (the weak $L^{p^{\prime}}$ space on $\mathbb{R}^{d}$ ) with $1 / p^{\prime}=1-$ $1 / d$ and $\left\|g_{\lambda}\right\|_{p^{\prime}, \mathrm{w}} \leq c_{d}$, where $\|\cdot\|_{p^{\prime}, \mathrm{w}}$ denotes the "pseudo" norm of $L_{\mathrm{w}}^{p^{\prime}}\left(\mathbb{R}^{d}\right)$ and $c_{d}$ is a constant independent of $\lambda>0$. By Cwikel's theorem [14, Section 3] and the condition $v \in$ $L^{d}\left(\mathbb{R}^{d}\right)$, which implies that $v_{\lambda} \in L^{d}\left(\mathbb{R}^{d}\right), v_{\lambda} R_{\lambda}$ is compact as an operator on $L^{2}\left(\mathbb{R}^{d}\right)$ and

$$
\mu_{n}\left(v_{\lambda} R_{\lambda}\right) \leq K_{1}\left\|v_{\lambda}\right\|_{L^{d}} n^{-1 / d}, \quad n \in \mathbb{N},
$$

where $K_{1}>0$ is a constant independent of $V, \lambda>0$ and $n \in \mathbb{N}$. Since $\operatorname{dim} \mathscr{K}<\infty$, it follows that $v_{\lambda} R_{\lambda}$ is compact also as an operator on $\mathscr{H}$. Let

$$
B_{\lambda}(x):= \begin{cases}\frac{V(x)}{v(x)} & \text { if } v(x)>\frac{\lambda}{4 m} \\ 0 & \text { otherwise. }\end{cases}
$$

Then $B_{\lambda}$ is bounded with $\left\|B_{\lambda}(x)\right\| \leq 1$. We have $V_{\lambda} R_{\lambda}=$ $B_{\lambda} v_{\lambda} R_{\lambda}$. Hence $V_{\lambda} R_{\lambda}$ is compact. This shows that all $T_{\lambda j}(j=$ $1,2,3,4,5)$ are compact.

In general, for all compact operators $A$ and bounded operators $B$ on a Hilbert space

$$
\mu_{n}(B A) \leq\|B\| \mu_{n}(A) .
$$

(e.g., see [15, Theorem 1.6].). Hence

$$
\mu_{n}\left(V_{\lambda} R_{\lambda}\right) \leq\left\|B_{\lambda}\right\| \mu_{n}\left(v_{\lambda} R_{\lambda}\right) \leq K_{1}\left\|v_{\lambda}\right\|_{L^{d}} n^{-1 / d} .
$$

Therefore

$$
\mu_{n}\left(T_{\lambda 1}\right) \leq\left\|H_{0} R_{\lambda}\right\| K_{1}\|v\|_{L^{d}} n^{-1 / d} \leq K_{1}\left\|v_{\lambda}\right\|_{L^{d}} n^{-1 / d} .
$$

Similarly one can show that $T_{\lambda 2}$, is compact and

$$
\mu_{n}\left(T_{\lambda 2}\right) \leq K_{1}\left\|v_{\lambda}\right\|_{L^{d}} n^{-1 / d}
$$


where we have use the fact that $\mu_{n}(A)=\mu_{n}\left(A^{*}\right)$ for all compact operators on a Hilbert space $[15,(1.3)]$.

As for $T_{\lambda 3}$, we write

$$
T_{\lambda 3}=m \alpha_{d+1} R_{\lambda} v_{\lambda}^{1 / 2} B_{\lambda} v_{\lambda}^{1 / 2} R_{\lambda}
$$

By the condition $v \in L^{d / 2}\left(\mathbb{R}^{d}\right), v_{\lambda}^{1 / 2} \in L^{d}\left(\mathbb{R}^{d}\right)$. Hence, Cwikel's theorem again, $v_{\lambda}^{1 / 2} R_{\lambda}$ is compact and

$$
\mu_{n}\left(v_{\lambda}^{1 / 2} R_{\lambda}\right) \leq K_{1}^{\prime}\left\|v_{\lambda}^{1 / 2}\right\|_{L^{d}} n^{-1 / d},
$$

where $K_{1}^{\prime}>0$ is a constant independent of $V$ and $\lambda>0$. We have

$$
\mu_{n}\left(T_{\lambda 3}\right) \leq m \mu_{n}\left(R_{\lambda} v_{\lambda}^{1 / 2} B_{\lambda} v_{\lambda}^{1 / 2} R_{\lambda}\right) .
$$

In general, for all compact operators $A$ and bounded operators $D$ on a Hilbert space,

$$
\begin{gathered}
\mu_{2 n+1}\left(A^{*} D A\right) \leq\|D\| \mu_{n+1}(A)^{2}, \\
\mu_{2 n}\left(A^{*} D A\right) \leq\|D\| \mu_{n}(A)^{2},
\end{gathered}
$$

where we have used the fact that, for all compact operators $A$ and $B$ on a Hilbert space,

$$
\mu_{n+k+1}(A B) \leq \mu_{n+1}(A) \mu_{k+1}(B), \quad n, k \geq 0 .
$$

Hence

$$
\begin{gathered}
\mu_{2 n+1}\left(T_{\lambda 3}\right) \leq m \mu_{n+1}\left(v_{\lambda}^{1 / 2} R_{\lambda}\right)^{2} \\
\leq m\left(K_{1}^{\prime}\right)^{2}\left\|v_{\lambda}^{1 / 2}\right\|_{L^{d}}^{2}(n+1)^{-2 / d}, \\
\mu_{2 n}\left(T_{\lambda 3}\right) \leq m \mu_{n}\left(v_{\lambda}^{1 / 2} R_{\lambda}\right)^{2} \leq m\left(K_{1}^{\prime}\right)^{2}\left\|v_{\lambda}^{1 / 2}\right\|_{L^{d}}^{2} n^{-2 / d},
\end{gathered}
$$

which imply that

$$
\mu_{n}\left(T_{\lambda 3}\right) \leq K_{2}^{\prime}\left\|v_{\lambda}^{1 / 2}\right\|_{L^{d}}^{2} n^{-2 / d}, \quad n \in \mathbb{N},
$$

where $K_{2}^{\prime}>0$ is a constant independent of $V, \lambda$, and $n$. Similarly we have

$$
\begin{gathered}
\mu_{n}\left(T_{\lambda 4}\right) \leq K_{2}^{\prime}\left\|v_{\lambda}^{1 / 2}\right\|_{L^{d}}^{2} n^{-2 / d}, \\
\mu_{n}\left(T_{\lambda 5}\right) \leq K_{3}^{\prime}\left\|v_{\lambda}\right\|_{L^{d}}^{2} n^{-2 / d}, \quad n \in \mathbb{N},
\end{gathered}
$$

where $K_{3}^{\prime}>0$ is a constant independent of $V, \lambda$ and $n$. Thus the desired results follow.

Theorem 30. Let $d \geq 3$, and suppose that the assumption of Lemma 25 holds and $\|V(\cdot)\| \in L^{d}\left(\mathbb{R}^{d}\right) \cap L^{d / 2}\left(\mathbb{R}^{d}\right)$. Let $\lambda \in$ $\left(0, m^{2}\right)$. Then, there exists a constant $C_{0}>0$ independent of $V$ and $\lambda$ such that

$$
N(\lambda, V) \leq C_{0} \int_{\|V(x)\|>\lambda / 4 m}\left(\|V(x)\|^{d / 2}+\|V(x)\|^{d}\right) d x .
$$

Proof. We need only to consider the case where $N$ := $N(\lambda, V) \geq 1$. Then there exists an $N$-dimensional subspace $E$ of $\mathscr{H}$ such that (106) holds for all $\psi \in E$. It is easy to see that $\left\|\left(V_{\lambda}-V\right) \phi\right\| \leq(\lambda / 4 m)\|\phi\|$, for all $\phi \in \mathscr{H}$. Let $\psi \in E$. Then, as in the proof of Theorem 28, we have $\left\|\left(H_{m}+V_{\lambda}\right) \psi\right\|^{2} \leq\left(m^{2}-(\lambda / 2)\right)\|\psi\|^{2}$, which is equivalent to the following inequality:

$$
\begin{gathered}
\left\|\left(-\Delta+\frac{\lambda}{2}\right)^{1 / 2} \psi\right\|^{2}+\left\langle H_{0} \psi, V_{\lambda} \psi\right\rangle+\left\langle V_{\lambda} \psi, H_{0} \psi\right\rangle \\
+m\left\langle\alpha_{d+1} \psi, V_{\lambda} \psi\right\rangle+m\left\langle V_{\lambda} \psi, \alpha_{d+1} \psi\right\rangle \\
+\left\|V_{\lambda} \psi\right\|^{2} \leq 0 .
\end{gathered}
$$

The subspace $F:=(-\Delta+\lambda / 2)^{1 / 2} E$ is also $N$-dimensional. Inequality (128) implies that, for all $\phi \in F$,

$$
\|\phi\|^{2} \leq\left\langle\phi, T_{\lambda} \phi\right\rangle
$$

where

$$
T_{\lambda}:=-\sum_{j=1}^{5} T_{\lambda j}
$$

By Lemma $29, T_{\lambda}$ is a compact self-adjoint operator on $\mathscr{H}$. Hence, by the Hilbert-Schmidt theorem, there exists a complete orthonormal system $\left\{\phi_{n}\right\}_{n=1}^{\infty}$ of $\mathscr{H}$ and a real sequence $\left\{t_{n}\right\}_{n=1}^{\infty}$ such that $T_{\lambda} \phi_{n}=t_{n} \phi_{n}$ and $\lim _{n \rightarrow \infty} t_{n}=0$. Using this fact, one sees that the number of eigenvalues $t_{n}$ of $T_{\lambda}$ with $t_{n} \geq 1$ is more than or equal to $\operatorname{dim} F=N$. Hence $\mu_{N}\left(T_{\lambda}\right) \geq 1$. Let $k$ be the largest natural number not exceeding $(N+4) / 5$. Then $5 k-4 \leq N$. Hence $1 \leq$ $\mu_{N}\left(T_{\lambda}\right) \leq \mu_{5 k-4}\left(T_{\lambda}\right)$. On the other hand, by a general fact on singular values of the sum of two compact operators (e.g., [15, Theorem 1.7]), we have

$$
\mu_{5 k-4}\left(T_{\lambda}\right) \leq \sum_{j=1}^{5} \mu_{k}\left(T_{\lambda j}\right)
$$

Using this fact and Lemma 29, we obtain

$$
1 \leq 2 C\left\|v_{\lambda}\right\|_{L^{d}} k^{-1 / d}+2 C\left\|v_{\lambda}^{1 / 2}\right\|_{L^{d}}^{2} k^{-2 / d}+C\left\|v_{\lambda}\right\|_{L^{d}}^{2} k^{-2 / d}
$$

We have $k \geq N / 5$. Hence

$$
1 \leq C^{\prime}\left(\left\|v_{\lambda}\right\|_{L^{d}} N^{-1 / d}+\left\|v_{\lambda}^{1 / 2}\right\|_{L^{d}}^{2} N^{-2 / d}+\left\|v_{\lambda}\right\|_{L^{d}}^{2} N^{-2 / d}\right),
$$

where $C^{\prime}>0$ is a constant independent of $V, \lambda$, and $N$. This implies that $N \leq C_{0}\left(\left\|v_{\lambda}^{1 / 2}\right\|_{L^{d}}^{d}+\left\|v_{\lambda}\right\|_{L^{d}}^{d}\right)$ with a constant $C_{0}$ independent of $V$ and $\lambda$. Thus (127) holds.

As in Corollaries 1.2 and 1.3 in [13], we have from Theorem 30 the following results. 
Corollary 31. Under the same assumption as in Theorem 30, the number $N(V)$ of eigenvalues of $H(V)$ in $(-m, m)$ is finite and

$$
N(V) \leq C_{0} \int_{\mathbb{R}^{d}}\left(\|V(x)\|^{d / 2}+\|V(x)\|^{d}\right) d x .
$$

Corollary 32. Suppose that the assumption of Theorem 30 holds. Let $\lambda_{j}(j=1, \ldots, N(V))$ be the eigenvalues of $H(V)$ in $(-m, m)$, counted with multiplicity, and let $\gamma>0$ be such that

$$
f_{\gamma}(V):=\int_{\mathbb{R}^{d}}\|V(x)\|^{\gamma}\left(\|V(x)\|^{d / 2}+\|V(x)\|^{d}\right) d x<\infty .
$$

Then, there exists a constant $C_{\gamma}>0$ such that

$$
\sum_{j=1}^{N(V)}\left(m^{2}-\lambda_{j}^{2}\right)^{\gamma} \leq C_{\gamma} f_{\gamma}(V)
$$

8.2. Applications. Now we apply the results in the preceeding section to the Dirac operator $H$. For $\lambda \in\left(0, m^{2}\right)$, we denote by $N(\lambda)$ the number of eigenvalues of $H$ in $\left(-\sqrt{m^{2}-\lambda}, \sqrt{m^{2}-\lambda}\right)$

Theorem 33. Let $d \geq 3$ and $\lambda \in\left(0, m^{2}\right)$. Suppose that the assumption of Theorem 26 holds. Let

$$
F_{M, \Phi}(x):=\|M(x)-m\|+2 m\left\|\sin \frac{\Phi(x)}{2}\right\|, \quad \text { a.e. } x \in \mathbb{R}^{d} \text {. }
$$

(i) If $F_{M, \Phi}(x) \leq \lambda / 4 m$, a.e. $x \in \mathbb{R}^{d}$, then $N(\lambda)=0$.

(ii) Suppose that $F_{M, \Phi} \in L^{d / 2}\left(\mathbb{R}^{d}\right) \cap L^{d}\left(\mathbb{R}^{d}\right)$. Then there exists a positive constant $C>0$ independent of $M, \Phi$, and $\lambda$ such that

$$
N(\lambda) \leq C \int_{F_{M, \Phi}(x)>\lambda / 4 m}\left(F_{M, \Phi}(x)^{d / 2}+F_{M, \Phi}(x)^{d}\right) d x<\infty .
$$
obeys

Moreover, the number $N_{0}$ of eigenvalues of $H$ in $(-m, m)$

$$
N_{0} \leq C \int_{\mathbb{R}^{d}}\left(F_{M, \Phi}(x)^{d / 2}+F_{M, \Phi}(x)^{d}\right) d x<\infty
$$

Proof. (i) We can write $H=H(V)$ with $V=\alpha_{d+1}\left(M e^{i \Phi}-m\right)$. Hence

$$
\begin{aligned}
\|V(x)\| & =\left\|M(x) e^{i \Phi(x)}-m\right\| \\
& \leq\|M(x)-m\|+m\left\|e^{i \Phi(x)}-1\right\|=F_{M, \Phi}(x) .
\end{aligned}
$$

Hence, the present assumption implies that $\|V(x)\| \leq \lambda / 4 m$ a.e. $x \in \mathbb{R}^{d}$. Hence, by Theorem $28, N(\lambda)=0$.

(ii) By (140) and the present assumption, $\|V(\cdot)\| \epsilon$ $L^{d}\left(\mathbb{R}^{d}\right) \cap L^{d / 2}\left(\mathbb{R}^{d}\right)$. Thus we can apply Theorem 30 to obtain (138). Inequality (139) follows from (138) or Corollary 31.

We have from Corollary 32 the following fact.
Corollary 34. Let $d \geq 3$. Suppose that the assumption of Theorem 26 and $F_{M, \Phi} \in L^{d / 2}\left(\mathbb{R}^{d}\right) \cap L^{d}\left(\mathbb{R}^{d}\right)$. Let $\lambda_{j}(j=$ $\left.1, \ldots, N_{0}\right)$ be the eigenvalues of $H$ in $(-m, m)$, counted with multiplicity, and let $\gamma>0$ be such that

$$
\begin{aligned}
& f_{\gamma}(M, \Phi) \\
& \quad:=\int_{\mathbb{R}^{d}} F_{M, \Phi}(x)^{\gamma}\left(F_{M, \Phi}(x)^{d / 2}+F_{M, \Phi}(x)^{d}\right) d x<\infty .
\end{aligned}
$$

Then, there exists a constant $C_{\gamma}>0$ such that

$$
\sum_{j=1}^{N_{0}}\left(m^{2}-\lambda_{j}^{2}\right)^{\gamma} \leq C_{\gamma} f_{\gamma}(M, \Phi) .
$$

We can also use Theorems 23 and 27 to obtain another upper bound for $N(\lambda)$. Let

$$
\begin{array}{r}
G_{M, \Phi}(x):=M(x)-m-\sum_{j=1}^{d} \alpha_{d+1} \alpha_{j} e^{i \Phi(x) / 2} E_{j}(x) \|, \\
\text { a.e. } x \in \mathbb{R}^{d} .
\end{array}
$$

Theorem 35. Let $d \geq 3$, and let $\lambda \in\left(0, m^{2}\right)$. Suppose that the assumption of Theorem 27 holds. Then one has the following.

(i) If $G_{M, \phi}(x) \leq \lambda / 4 m$ for a.e. $x \in \mathbb{R}^{d}$, then $N(\lambda)=0$.

(ii) Suppose that $G_{M, \Phi} \in L^{d / 2}\left(\mathbb{R}^{d}\right) \cap L^{d}\left(\mathbb{R}^{d}\right)$. Then (138) and (139) with $F_{M, \Phi}$ replaced by $G_{M, \Phi}$ hold.

Proof. By Theorem 23, $N(\lambda)$ is equal to the number of eigenvalues of $H^{\prime}$ in $\left(-\sqrt{m^{2}-\lambda}, \sqrt{m^{2}-\lambda}\right)$. One can write $H^{\prime}=H_{m}+V$ with $V:=\alpha_{d+1}\left(M-m-\sum_{j=1}^{d} \alpha_{d+1} \alpha_{j} A_{j}\right)$. We have $\|V(x)\|=G_{M, \Phi}(x)$. Thus, in the same way as in the proof of Theorem 30, we obtain the desired results.

Theorem 35 implies the following result as in Corollary 34.

Corollary 36. Let $d \geq 3$. Suppose that the assumption of Theorem 27 holds and $G_{M, \Phi} \in L^{d / 2}\left(\mathbb{R}^{d}\right) \cap L^{d}\left(\mathbb{R}^{d}\right)$. Then (142) with $F_{M, \Phi}$ replaced by $G_{M, \Phi}$ holds for all $\lambda>0$ such that $\int_{\mathbb{R}^{d}} G_{M, \Phi}(x)^{\gamma}\left(G_{M, \Phi}(x)^{d / 2}+G_{M, \Phi}(x)^{d}\right) d x<\infty$.

\section{Acknowledgment}

Asao Arai is supported by the Grant-in-Aid 24540154 for Scientific Research from JSPS.

\section{References}

[1] N. Sawado and N. Shiiki, "Degeneracy of the quarks, shell structure in the chiral soliton," Nuclear Physics A, vol. 739, no. 1-2, pp. 89-108, 2004.

[2] H. Kalf and O. Yamada, "Essential self-adjointness of $n$ dimensional Dirac operators with a variable mass term," Journal of Mathematical Physics, vol. 42, no. 6, pp. 2667-2676, 2001. 
[3] A. Arai, K. Hayashi, and I. Sasaki, "Spectral properties of a Dirac operator in the chiral quark soliton model," Journal of Mathematical Physics, vol. 46, no. 5, Article ID 052306, 12 pages, 2005.

[4] A. Arai, "Mathematical analysis of a generalized chiral quark soliton model," Symmetry, Integrability and Geometry. Methods and Applications, vol. 2, paper 018, 12 pages, 2006.

[5] T. Miyao, "Nonrelativistic limit of the abstract chiral quark soliton model and confining effects," Journal of Operator Theory, vol. 57, no. 2, pp. 429-441, 2007.

[6] B. Thaller, The Dirac Equation, Texts and Monographs in Physics, Springer, Berlin, Germany, 1992.

[7] S. Pedersen, "Anticommuting selfadjoint operators," Journal of Functional Analysis, vol. 89, no. 2, pp. 428-443, 1990.

[8] F.-H. Vasilescu, "Anticommuting selfadjoint operators," Revue Roumaine de Mathématiques Pures et Appliquées, vol. 28, no. 1, pp. 76-91, 1983.

[9] M. Reed and B. Simon, Methods of Modern Mathematical Physics. vol. 1. Functional Analysis, Academic Press, New York, NY, USA, 1972.

[10] M. Reed and B. Simon, Methods of Modern Mathematical Physics. vol. 4. Analysis of Operators, Academic Press, New York, NY, USA, 1978.

[11] M. Reed and B. Simon, Methods of Modern Mathematical Physics. vol. 2. Fourier Analysis, Self-Adjointness, Academic Press, New York, NY, USA, 1975.

[12] T. Kato, Perturbation Theory for Linear Operators, Springer, Berlin, Germany, 2nd edition, 1976.

[13] C. Cancelier, P. Lévy-Bruhl, and J. Nourrigat, "Remarks on the spectrum of Dirac operators," Acta Applicandae Mathematicae, vol. 45, no. 3, pp. 349-364, 1996.

[14] M. Cwikel, "Weak type estimates for singular values and the number of bound states of Schrödinger operators," vol. 106, no. 1, pp. 93-100, 1977.

[15] B. Simon, Trace Ideals and Their Applications, vol. 120 of Mathematical Surveys and Monographs, American Mathematical Society, Providence, RI, USA, Second edition, 2005. 


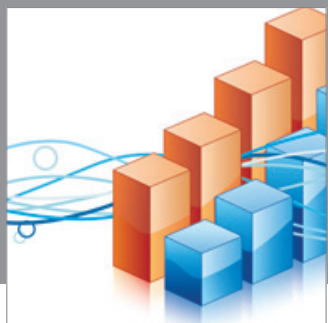

Advances in

Operations Research

mansans

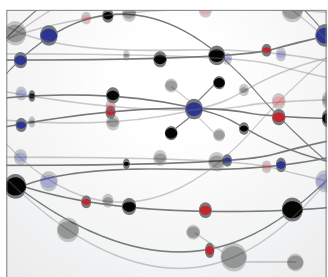

The Scientific World Journal
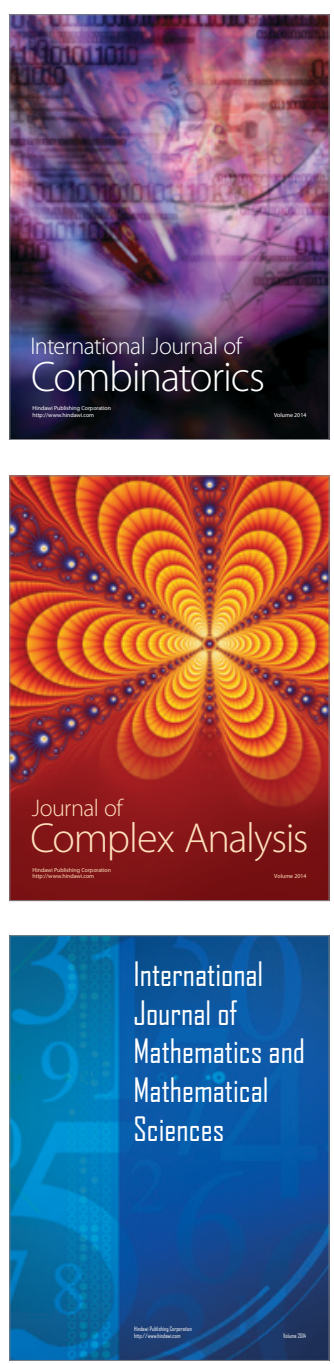
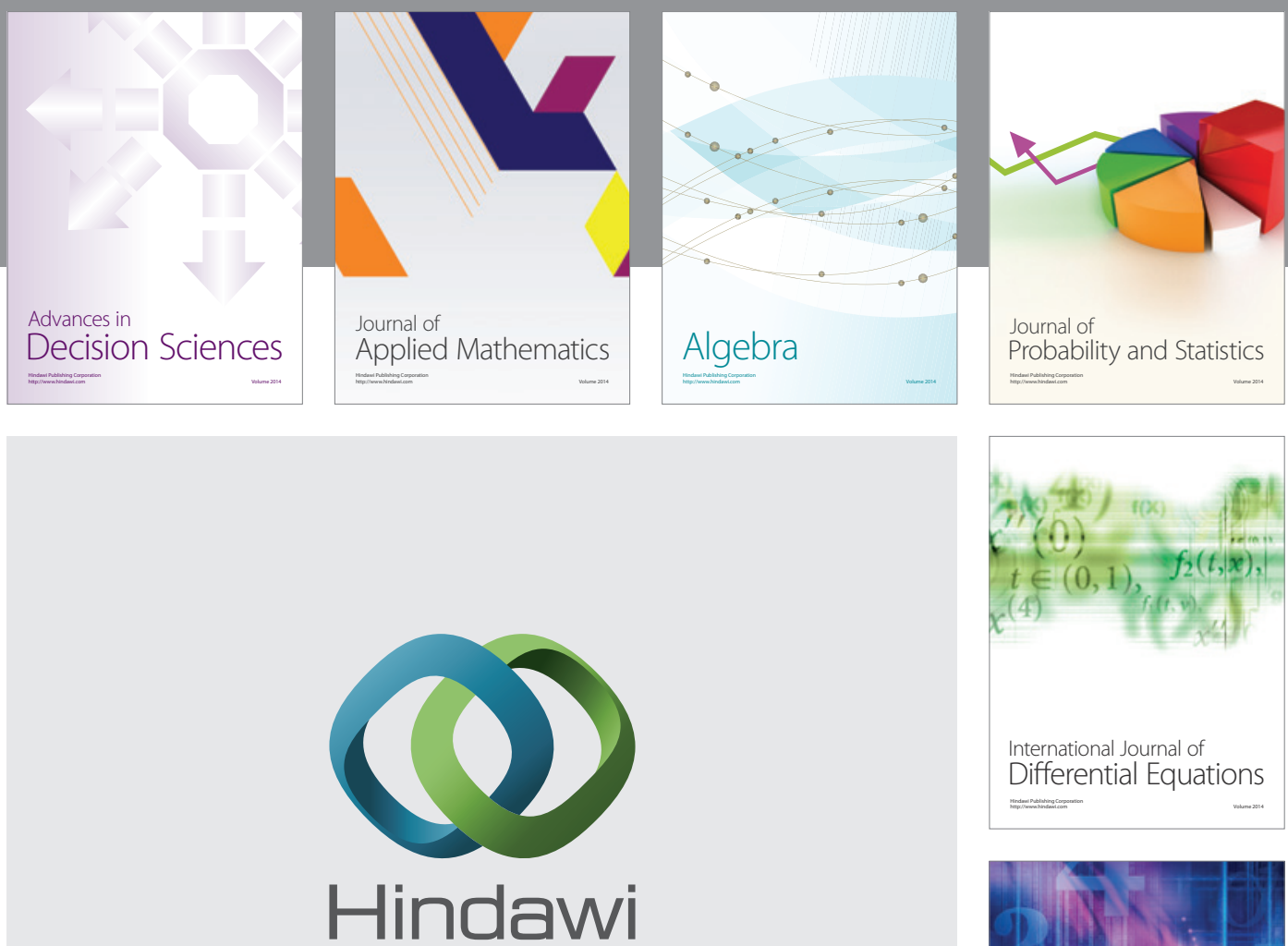

Submit your manuscripts at http://www.hindawi.com
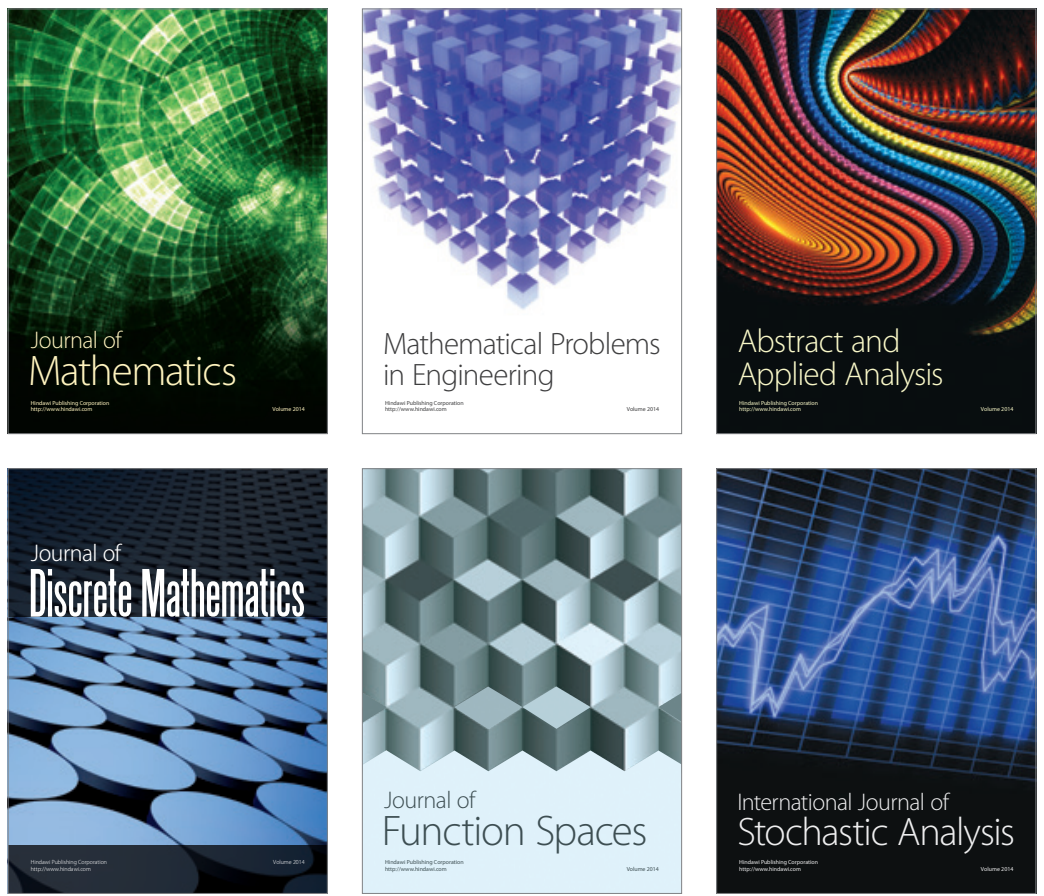

Journal of

Function Spaces

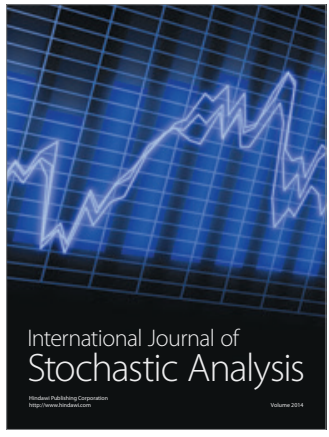

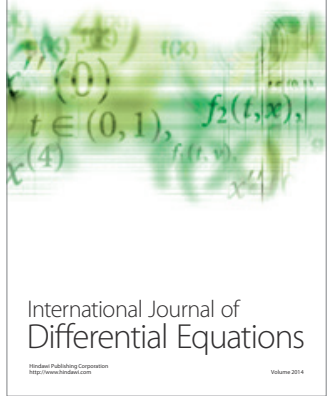
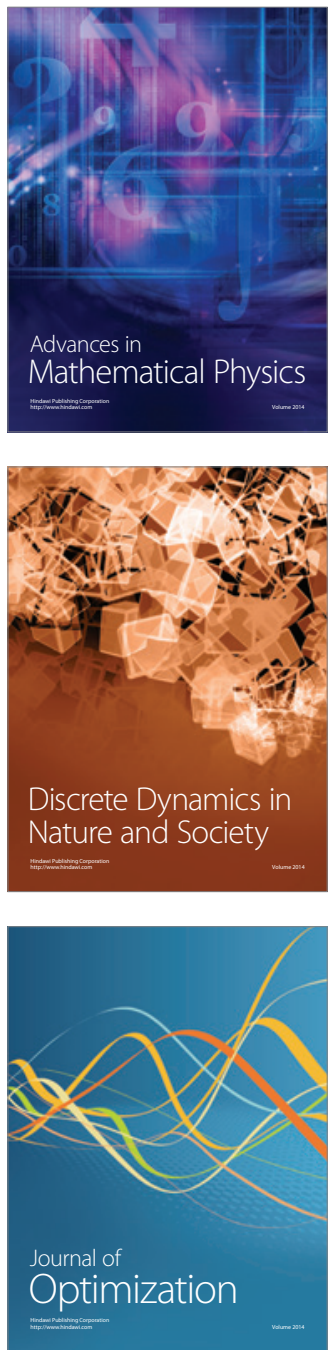